\title{
Neighbor-locating coloring: graph operations and extremal cardinalities
}

\author{
Carmen Hernando, Mercè Mora, Ignacio M Pelayo ${ }^{2}$ \\ Dept. de Matemáticas, Universitat Politècnica de Catalunya, Barcelona, Spain \\ Liliana Alcón, Marisa Gutierrez ${ }^{3}$ \\ Centro de Matemática, Universidad Nacional de La Plata, La Plata, Argentina
}

\begin{abstract}
A $k$-coloring of a graph $G=(V, E)$ is a $k$-partition $\Pi=\left\{S_{1}, \ldots, S_{k}\right\}$ of $V$ into independent sets, called colors. A $k$-coloring is called neighbor-locating if for every pair of vertices $u, v$ belonging to the same color $S_{i}$, the set of colors of the neighborhood of $u$ is different from the set of colors of the neighborhood of $v$. The neighbor-locating chromatic number, $\chi_{N L}(G)$, is the minimum cardinality of a neighbor-locating coloring of $G$.

In this paper, we examine the neighbor-locating chromatic number for various graph operations: the join, the disjoint union and Cartesian product. We also characterize all connected graphs of order $n \geq 3$ with neighbor-locating chromatic number equal either to $n$ or to $n-1$ and determine the neighbor-locating chromatic number of split graphs.
\end{abstract}

Keywords: Coloring, location, neighbor-location, complete multipartite graph, join graph, split graph, disjoint union, Cartesian product. 


\section{Introduction}

Domination and location in graphs are two important concepts that have received a lot of attention, usually separately, but sometimes also both together. There are mainly two types of location, the metric location and the neighbor location. In this work, we are interested in the neighbor location, and we study this concept in the particular context of a special kind of vertex partitions, called colorings.

In [6], G. Chartrand, E. Salehi and P. Zhang, brought the concept of metric-location to the ambit of vertex partitions. Metric-location and domination, in the context of vertex partitions, is studied in [7]. In [3], there were introduced the so-called locating colorings considering partitions formed by independents sets.

Neighbor location in sets was introduced by P. Slater in [8]. In [2], bounds of this parameter are given. In [1], merging the concepts of papers [3,8], there were introduced the so-called neighbor-locating colorings and the neighborlocating chromatic number, studying this parameter in some basic families of graphs.

In this work, we examine the neighbor-locating chromatic number for various graph operations. In Section 2, we study the join graph and all connected graphs with order $n$ and neighbor-locating chromatic number equal to $n$ are characterized.

The neighbor-locating chromatic number of all connected split graphs is determined in Section 3. The disjoint union and the Cartesian product are studied in Section 4. Finally, in Section 5, all connected graphs of order $n \geq 3$ with neighbor-locating chromatic number $n-1$ are given.

We introduce now some basic terminology. The distance between vertices $v, w \in V$ of a graph $G=(V, E)$ is denoted by $d(v, w)$. The diameter of $G$ is $\operatorname{diam}(G)=\max \{d(v, w): v, w \in V\}$. The distance $d(v, S)$ between a vertex $v \in V$ and a set of vertices $S \subseteq V$ is $d(v, S)=\min \{d(v, w): w \in S\}$. Let $\Pi=\left\{S_{1}, \ldots, S_{k}\right\}$ be a partition of $V$. If all the parts of $\Pi$ are independent sets, then we say that $\Pi$ is a coloring of $G$ and each part is a color.

A coloring $\Pi=\left\{S_{1}, \ldots, S_{k}\right\}$ is called a (metric-)locating coloring, an MLcoloring for short, if for every $i \in\{1, \ldots, k\}$ and for every pair of distinct

1 Research partially supported by projects Gen. Cat. DGR 2017SGR1336 and MTM201563791-R 276 (MINECO/FEDER), H2020-MSCA-RISE-2016-734922 CONNECT and CONICET

2 Email: carmen.hernando@upc.edu, merce.mora@upc.edu, ignacio.m.pelayo@upc.edu

3 Email: liliana@mate.unlp.edu.ar, marisa@mate.unlp.edu.ar 
vertices $u, v \in S_{i}$, there exists $j \in\{1, \ldots, k\}$ such that $d\left(u, S_{j}\right) \neq d\left(v, S_{j}\right)$. The (metric-)locating-chromatic number $\chi_{L}(G)$ is the minimum cardinality of an ML-coloring of $G$.

A coloring $\Pi=\left\{S_{1}, \ldots, S_{k}\right\}$ is called a neighbor-locating coloring, an NLcoloring for short, if for every $i \in\{1, \ldots, k\}$ and for every pair of not isolated distinct vertices $u, v \in S_{i}$, there exists $j \in\{1, \ldots, k\}$ such that either $d\left(u, S_{j}\right)=1$ and $d\left(v, S_{j}\right) \neq 1$ or $d\left(u, S_{j}\right) \neq 1$ and $d\left(v, S_{j}\right)=1$ and there may be at most one isolated vertex for each color. The neighbor-locating chromatic number $\chi_{N L}(G)$ is the minimum cardinality of an NL-coloring of $G$. For undefined basic concepts of graph theory we refer the reader to the text [5].

\section{Join graphs}

A graph $G=(V, E)$ is said to be a join graph if it is the join $G_{1} \vee G_{2}$ of two graphs $G_{1}=\left(V_{1}, E_{1}\right)$ and $G_{2}=\left(V_{2}, E_{2}\right)$, i.e., if $V=V_{1} \cup V_{2}$ and $E=E_{1} \cup E_{2} \cup E^{\prime}$, where $E^{\prime}=\left\{v_{1} v_{2}: v_{1} \in V_{1}, v_{2} \in V_{2}\right\}$.

As a straightforward consequence of this definition, the following properties hold.

Proposition 2.1 Let $G_{1}, G_{2}$ and $G_{3}$ be three graphs.

(i) $G_{1} \vee G_{2}$ is a connected graph of diameter at most 2.

(ii) $G_{1} \vee\left(G_{2} \vee G_{3}\right) \cong\left(G_{1} \vee G_{2}\right) \vee G_{3}$.

Theorem 2.2 Let $G$ and $H$ be two graphs. Then,

$$
\chi_{N L}(G \vee H)=\chi_{N L}(G)+\chi_{N L}(H) .
$$

Let $r, n_{1}, \ldots, n_{r}, n$ integers such that $2 \leq r, 1 \leq n_{1} \leq \ldots \leq n_{r}$ and $n=$ $n_{1}+\ldots+n_{r}$. Then, the complete $r$-partite graph $K_{n_{1}, \ldots, n_{r}}$ is the graph $\overline{K_{n_{1}}} \vee$ $\ldots \vee \overline{K_{n_{r}}}$, where $\overline{K_{n_{i}}}$ denotes the empty graph of order $n_{i}$, i.e., the complement of the complete graph of order $n_{i}$. Notice that complete graphs, complete bipartite graphs and stars are some examples of complete multipartite graphs.

Theorem 2.3 Let $G$ be a connected graph of order $n \geq 3$. Then, $\chi_{N L}(G)=n$ if and only if $G$ is a complete multipartite graph.

\section{$3 \quad$ Split graphs}

A split graph is a graph such that the vertices can be partitioned into a clique and an independent set. When every vertex in the independent set is adjacent 
to every vertex in the clique it is said to be a complete split graph. Observe that complete split graphs are examples of complete multipartite graphs that we just studied in the previous section. We have taken a step further and we have studied the neighbor-locating chromatic number in general split graphs.

For any connected split graph $G=(V, E)$ we can assume that there are two subsets $U$ and $W$ of $V$ in such a way that (i) $V=U \cup W, U \cap W=\emptyset$; (ii) $G[U]$ is a complete graph; (iii) $G[W]$ is an empty graph; (iv) $W$ is a maximal independent set, i.e., for each vertex $u \in U$ there exists a vertex $w \in W$ such that $u w \in E$.

For every $X \subseteq U$, we consider the set $\mathcal{P}(X)=\{w \in W: N(w)=X\}$ and we denote $\rho(G)=\max \{|X|+|\mathcal{P}(X)|: X \subseteq U\}$. Observe that $\rho(G) \geq|U|$.

Theorem 3.1 If $G=(V, E)$ is a connected split graph, then

$$
\chi_{N L}(G)=\left\{\begin{array}{lr}
\rho(G), & \text { if } \mathcal{P}(X)=\emptyset \text { for all } X \subseteq U \text { s.t. }|X|=|U|-1 \\
\max (|U|+1, \rho(G)), & \text { if } \mathcal{P}(X) \neq \emptyset \text { for some } X \subseteq U \text { s.t. }|X|=|U|-1
\end{array}\right.
$$

Remark 3.2 The value of the neighbor-locating chromatic number obtained for general split graphs fits with some known results, such as $\chi_{N L}\left(S_{1, n-1}\right)=n$ or $\chi_{N L}(G)=n$, if $G$ is a complete split graph.

\section{Disjoint union and Cartesian product}

The disjoint union, $G+H$, of graphs $G$ and $H$ such that $V(G) \cap V(H)=\emptyset$ is a graph where the vertex set is $V(G) \cup V(H)$ and the edge set is $E(G) \cup E(H)$. In this section, we study the relationship between $\chi_{N L}(G+H), \chi_{N L}(G)$ and $\chi_{N L}(H)$.

Theorem 4.1 Let $G, H$ be two graphs. If $\chi_{N L}(G)=k$ and $\chi_{N L}(H)=h$, with $k \geq h$, then $k \leq \chi_{N L}(G+H) \leq k+h$.

Theorem 4.2 Let $k, h$ integers numbers where $k \geq h$. Then, for every $l \in$ $[k, k+h]$, there exist graphs $G$ and $H$ such that $\chi_{N L}(G)=k, \chi_{N L}(H)=h$ and $\chi_{N L}(G+H)=l$.

Theorem 4.3 Let $G, H$ be two graphs and let $\chi_{N L}(G)=k, \chi_{N L}(H)=h$. If $H$ has no isolated vertices, then $k \leq \chi_{N L}(G+H) \leq k+h-1$.

Theorem 4.4 Let $G$ be a graph and let $\chi_{N L}(G)=k$. If $G$ contains a universal vertex, then $\chi_{N L}(G+G) \leq k+1$.

The Cartesian product, $G \square H$, of graphs $G$ and $H$ is a graph such that the vertex set is $V(G) \times V(H)$ and two vertices $\left(u, u^{\prime}\right)$ and $\left(v, v^{\prime}\right)$ are adjacent 
in $G \square H$ if and only if either $u=v$ and $u^{\prime}$ is adjacent to $v^{\prime}$ in $H$, or $u^{\prime}=$ $v^{\prime}$ and $u$ is adjacent to $v$ in $G$. Now, we study the relationship between $\chi_{N L}(G \square H), \chi_{N L}(G)$ and $\chi_{N L}(H)$.

Theorem 4.5 Let $G, H$ be connected graphs, then

$$
\chi_{N L}(G \square H) \leq \chi_{N L}(G) \chi_{N L}(H) .
$$

Note that for $G \cong H \cong K_{2}$ the above inequality is attained.

Theorem 4.6 If $G$ is a graph with a universal vertex and $H$ is a connected graph, then $\chi_{N L}(G \square H) \leq \chi_{N L}(G)+|V(H)|-1$.

\section{$5 \quad$ Extremal graphs: case $\chi_{N L}(G)=n-1$}

In Section 2, we have characterized all the connected graphs with neighborlocating chromatic number equal to the order of the graph. Now, we determine the connected graphs of order $n$ and neighbor-locating chromatic number equal to $n-1$.

- Let $\mathcal{H}$ denote the set of all connected graphs $G$ or order $n \geq 3$ such that $H \cong G-v$ is a complete multipartite graph for some vertex $v$ of $G$.

- Let $V_{1}, V_{2}, \ldots, V_{k}, k \geq 2$, the partite sets of $H$, where $\left|V_{i}\right|=n_{i}$ and $a_{i}=\left|N(v) \cap V_{i}\right|(1 \leq i \leq k)$.

- Let $\mathcal{F}$ denote the set of all graphs $G \in \mathcal{H}$ that satisfy al least one of the following properties:

(1) For each integer $i \in\{1, \ldots, k\}$, we have $a_{i} \in\left\{0, n_{i}\right\}$ and there are at least two distinct integers $j, j^{\prime} \in\{1, \ldots, k\}$ for which $a_{j}=a_{j^{\prime}}=0$.

(2) There is exactly one integer $j \in\{1, \ldots, k\}$ such that $0<a_{j}<n_{j}$, and $a_{j}=n_{j}-1$ for this integer $j$.

- Let $\mathcal{G}$ denote the set of all graphs $G=G_{n} \vee 2 K_{2}$ where $G_{n}$ is a complete multipartite graph $G_{n}$ of order $n-4 \geq 1$.

Theorem 5.1 Let $G$ be a connected graph of order $n \geq 5$. Then, $\chi_{N L}(G)=$ $n-1$ if and only if $G \in \mathcal{F} \cup \mathcal{G}$.

The proof of this Theorem is based on the following lemmas and previous results.

Theorem 5.2 ([4]) Let $G$ be a connected graph of order $n \geq 4$. Then, $\chi_{L}(G)=n-1$ if and only if $G \in \mathcal{F} \cup \mathcal{G}$.

If a graph $G_{1}$ is an induced subgraph of a graph $G_{2}$, we write $G_{1} \prec G_{2}$. 
Lemma 5.3 Let $G$ be a connected graph of order $n \geq 5$. If $\chi_{N L}(G)=n-1$ and $2 K_{2} \prec G$, then $G \in \mathcal{G}$.

Lemma 5.4 ([4]) Let $G$ be a connected graph of order $n \geq 4$. If $\chi_{L}(G)=$ $n-1$ and $2 K_{2} \nprec G$, then $G \in \mathcal{F}$.

Lemma 5.5 Let $G$ be a graph of order $n \geq 5$ and diameter 3 such that $2 K_{2} \nprec$ $G$. If $\chi_{N L}(G)=n-1$, then $\chi_{L}(G)=n-1$.

Lemma 5.6 Let $G$ be a connected graph of order $n \geq 3$.

(i) If $\operatorname{diam}(G) \leq 2$, then $\chi_{L}(G)=\chi_{N L}(G)$.

(ii) If $\operatorname{diam}(G) \geq 4$, then $\chi_{N L}(G) \leq n-2$.

\section{References}

[1] L. Alcón, M. Gutiérrez, C. Hernando, M. Mora and I. M. Pelayo: Neighborlocating colorings in graphs. Preprint.

[2] J. Cáceres, C. Hernando, M. Mora, I. M. Pelayo and M. L. Puertas: Locatingdominating codes: bounds and extremal cardinalities. Appl. Math. Comput., 220 (2013), 38-45.

[3] G. Chartrand, D. Erwin, M. A. Henning, P. J. Slater and P. Zhang: The locatingchromatic number of a graph. Bull. Inst. Combin. Appl., 36 (2002), 89-101.

[4] G. Chartrand, D. Erwin, M. A. Henning, P. J. Slater and P. Zhang: Graphs of order $n$ with locating-chromatic number $n-1$. Discrete Math., 269 (2003), $65-79$.

[5] G. Chartrand, L. Lesniak, P. Zhang: Graphs and Digraphs, (5th edition), CRC Press, Boca Raton, Florida, (2011).

[6] G. Chartrand, E. Salehi and P. Zhang: The partition dimension of a graph. Aequationes Mathematicae, 59 (2000), 45-54.

[7] C. Hernando, M. Mora and I. M. Pelayo: Metric-locating-dominating partitions in graphs. Submitted. (https://arxiv.org/abs/1711.01086).

[8] P. J. Slater, Dominating and reference sets in a graph, J. Math. Phys. Sci., 22 (1988), 445-455. 